\title{
Estratégias grupais voltadas aos familiares de pessoas com necessidades decorrentes do uso de substâncias psicoativas: uma revisão narrativa*
}

\section{Group strategies oriented to family members of people with needs resulting from the use of psychoactive substances: a narrative review}

\author{
Helton Alves de Lima ${ }^{1}$, Elisabete Ferreira Mângia ${ }^{2}$
}

http://dx.doi.org/10.11606/issn.2238-6149.v26i2p294-300

\begin{abstract}
Lima HA, Mângia EF. Estratégias grupais voltadas aos familiares de pessoas com necessidades decorrentes do uso de substâncias psicoativas: uma revisão narrativa. Rev Ter Ocup Univ São Paulo. 2015 maio-ago.;26(2):294-300.
\end{abstract}

RESUMO: O estudo apresenta e discute tendências de estratégias grupais voltadas aos familiares de pessoas que fazem uso prejudicial de substâncias psicoativas atendidas em serviços de saúde mental, identifica as perspectivas teóricas adotadas, suas modelagens e papel no contexto da organização do processo de cuidado. Foi desenvolvida a revisão narrativa a partir da leitura de artigos e capítulos de livros em língua portuguesa especializados. Foram consultadas as bases BVS, BVS Psicologia, SciELO e Lilacs, e selecionados seis artigos e três capítulos de livros. A partir dos conteúdos identificados, definiu-se os seguintes campos temáticos: contexto de desenvolvimento da estratégia grupal, perspectiva teórica, modelagem das estratégias e o papel das estratégias grupais no cuidado aos familiares. As principais ancoragens teóricas e técnicas identificadas foram as estratégias psicoeducativas e as de apoio e ampliação de rede sócio relacional. Discute-se a importância e as potencialidades de tais estratégias na atenção às famílias, como estas respondem às necessidades de ampliação do acesso das famílias aos serviços, ao suporte social e emocional, e à compreensão sobre uso prejudicial de substâncias psicoativas e seus tratamentos. A escolha e a adoção das metodologias das estratégias grupais devem acontecer por meio de processos de reflexão das equipes, considerando as necessidades apresentadas pelos familiares. Deve também pautar-se pelo reconhecimento das histórias de vida e singularidades dos sujeitos, evitar o olhar exclusivamente intervencionista sobre a doença e priorizar a produção de vínculos e autonomia.

DESCRITORES: Prática de grupo/tendências; Família; Transtornos relacionados ao uso de substâncias; Assistência à saúde mental.

Lima HA, Mângia EF. Group strategies oriented to family members of people with needs resulting from the use of psychoactive substances: a narrative review. Rev Ter Ocup Univ São Paulo. 2015 May-Aug.;26(2):294-300.

ABSTRACT: The study presents and discusses trends of group strategies regarding the families of people who make harmful use of psychoactive substances treated in mental health services and identifies the theoretical perspectives adopted, their modeling and role in the organization of care process. The narrative review was developed from reading articles and chapters of specialized books in Portuguese. The BVS, BVS Psychology, SciELO and Lilacs databases were consulted, and six articles and three book

\footnotetext{
* Trabalho de conclusão do curso de Residência Multiprofissional em Saúde Mental com ênfase em Dependência Química, apresentado pelo primeiro autor à Faculdade de Medicina da USP São Paulo, sob orientação da segunda autora.

1. Psicólogo, formação em Saúde Coletiva (IS-SESSP) e Saúde Mental com ênfase em Dependência Química pela Faculdade de Medicina da Universidade de São Paulo - FMUSP. E-mail: lima.helton@gmail.com.

2. Prof ${ }^{a} \operatorname{Dr}^{\mathrm{a}}$ do Departamento de Fisioterapia, Fonoaudiologia e Terapia Ocupacional da Faculdade de Medicina da Universidade de São Paulo - FMUSP. E-mail: mangeli@usp.br.

Endereço para correspondência: Elisabete F. Mângia. FOFITO. Rua Cipotânea, 51 - Cidade Universitária. São Paulo, SP, Brasil. CEP: 05360-000. E-mail: mangeli@usp.br.
} 
chapters were selected. From the identified contents the following thematic fields were defined: the context of group strategy development, theoretical perspective, modeling of strategies and the role of group strategies in health care to these families. The main theoretical and technical anchorages identified were the psychoeducational strategies and the support and expansion of social relational network. It discusses the importance and potential of these strategies in the care of families, how they respond to the needs of expansion of access of the families to the services, to social and emotional support and to understanding the

\section{INTRODUÇÃO}

$\mathrm{A}$ atenção em saúde voltada aos familiares de pessoas com necessidades decorrentes do uso de substâncias psicoativas, no âmbito dos serviços de saúde mental, ancora-se em dimensões políticas, históricas, técnicas e éticas que compõem o paradigma da atenção psicossocial*. No Brasil, a Política de Saúde Mental compreende a atenção aos familiares de pessoas em situação de vulnerabilidade e sofrimento psíquico no contexto do resgate dos direitos de cidadania, que deve ser orientada pelos princípios da clínica ampliada.

Historicamente, a atenção psiquiátrica promoveu a exclusão e a violação de direitos de milhares de indivíduos, reclusos nos hospitais psiquiátricos e manicômios judiciários. Reduzidas à crise e à sintomatologia, as pessoas com transtornos mentais eram afastadas de seus contextos de vida, o que intensificava a fragilização e o rompimento dos vínculos e gerava efeitos negativos na manutenção das relações interpessoais e comunitárias ${ }^{1,2}$. Com a Reforma Psiquiátrica, o cuidado em saúde mental assumiu as diretrizes da integralidade e da intersetorialidade na construção dos projetos terapêuticos singulares, e foi deslocado para os territórios socioeconômicos e existenciais dos indivíduos e suas famílias, passando a fazer parte do cotidiano dos familiares nas diversas ações relativas à produção do cuidado e de respostas as suas necessidades ${ }^{3}$.

A discussão acerca do papel da família no cuidado considera o potencial do grupo familiar como uma instituição protetora ${ }^{4}$. Analisa os impactos objetivos e subjetivos do adoecimento de um de seus membros que geram sobrecarga e necessidade de cuidado pela rede de harmful use of psychoactive substances and its treatments. The choice and adoption of methodologies of group strategies should happen through the thinking processes of the teams, considering the needs presented by the family. It should also be based on the recognition of their life histories and the uniqueness of the individual, avoiding an exclusively interventionist look at the disease and prioritizing the production of bonds and autonomy.

KEYWORDS: Group practice/trends; Family; Substance-related disorders; Mental health assistance.

atenção ${ }^{3}$, e busca construir relações de parceria e suporte para o restabelecimento da saúde de todo grupo familiar ${ }^{5}$.

A Política de Atenção Integral aos Usuários de Álcool e outras Drogas prevê o desenvolvimento de ações que incluam os familiares, destacando-se aquelas que visam reduzir fatores de segregação sociofamiliar, prioriza o desenvolvimento de fatores de proteção e redução de riscos, assim como o cuidado dirigido aos familiares ${ }^{6}$. Tais ações colocam a família como parceira e ampliam seu papel no cuidado psicossocial e desenvolvimento de projetos terapêuticos singulares? .

A identificação do impacto dos problemas associados ao consumo de álcool e outras drogas na vida de familiares, cuidadores e outras pessoas significativas, impõe a reflexão acerca das necessidades de saúde para além daquelas identificadas no usuário. Estudo de revisão promovido pelo governo britânico identificou quatro áreas de impacto geradoras de necessidades específicas na vida de familiares. São elas: a) saúde física e psicológica: em decorrência da ansiedade, culpa, solidão e outros sentimentos; b) relações familiares: correlacionadas às mudanças de papéis entre os membros, falhas na comunicação, rupturas e conflitos; c) financeiros $e$ ocupacionais: decorrentes de estratégias para obtenção das substâncias, dívidas de drogas, despesas de tratamento, entre outras; d) vida social: decorrentes do isolamento em relação à família e amigos, temor em deixar o usuário sozinho, receio de ser alvo de críticas do grupo social, e pouca motivação para o lazer ${ }^{8}$.

$\mathrm{Na}$ perspectiva da integralidade ${ }^{9}$, o atendimento às necessidades de saúde tem caráter estratégico; essas devem ser acolhidas pelas equipes, o que envolve esforço interdisciplinar de articulação de saberes, e respondidas por meio de tecnologias construídas no contexto da relação

\footnotetext{
* Para Amarante ${ }^{1}$, a atenção psicossocial não deve ser confundida com modelo ou sistema fechado, mas como processo social complexo e cuja transformação é contínua e aberta a novos problemas e enfrentamentos. Tem nos serviços de saúde mental seu espaço estratégico de acolhimento, cuidado e trocas sociais.)
} 
estabelecida com usuários, familiares e comunidade. Nesse processo, Merhy ${ }^{10}$ alerta para o papel fundamental das tecnologias leves, que envolvem as relações interpessoais e visam à produção de vínculos, autonomia e corresponsabilização; e leve-duras, que compreendem os saberes que estruturam e organizam as ações dos profissionais, o conhecimento aplicado e relativo a um saber fazer específico ${ }^{10,11}$.

Os grupos de ajuda e suporte mútuos ${ }^{12}$ são estratégias capazes de induzir o protagonismo e empoderamento de usuários e familiares, além de componentes-chave do processo de cuidado e reabilitação psicossocial. Os modelos de funcionamento e organização do trabalho técnico nas redes de atenção psicossocial preconizam a organização e oferta de diversas modalidades de estratégias grupais, tais como: grupos e oficinas terapêuticas, educativas e de geração de renda, de familiares, assembleias, entre outras $^{13}$

A reflexão sobre possibilidades e limites das estratégias grupais na saúde mental se coloca como fator de qualificação dos processos de cuidado aos quais estão articuladas. Por considerar a importância desse tema e buscando subsídios para seu desenvolvimento, este estudo visou conhecer as estratégias grupais voltadas aos familiares de pessoas que fazem uso prejudicial de álcool e outras drogas. Assim, buscou descrever e analisar as estratégias grupais desenvolvidas e relatadas na literatura e, mais especificamente, identificar e refletir sobre as perspectivas teóricas adotadas, sua modelagem e seu papel na organização do processo de cuidado dos serviços.

\section{METODOLOGIA}

A reflexão apresentada está ancorada no desenvolvimento de uma revisão narrativa, que consistiu na avaliação teórica, contextual e não sistemática de publicações selecionadas ${ }^{14}$, de acordo com objetivos traçados pelo pesquisador ${ }^{15}$. Foram selecionados artigos nacionais sobre estratégias grupais para familiares, no período de 1994 a 2014 a partir das bases da BVS (Biblioteca Virtual em Saúde) e BVS Psicologia. Utilizou-se nove conjuntos de combinações de descritores, a saber: grupo and familia and dependência química; grupo $e$ família $e$ toxicodependência; grupo $e$ apoio $e$ família $e$ dependência química; grupo $e$ apoio $e$ família $e$ toxicomania; grupo $e$ apoio $e$ família $e$ droga; grupo $e$ apoio $e$ família $e$ álcool; grupo $e$ multifamiliar $e$ dependência química; transtornos relacionados ao uso de substâncias psicoativas $e$ grupo $e$ familia; ajuda a famílias com filhos dependentes.
Foram encontrados 288 artigos, dos quais seis foram selecionados por se relacionarem com os objetivos, e incluídos sete livros sobre tratamento para usuários de substâncias psicoativas que discutem estratégias grupais voltadas para familiares, publicados no período de 1994 a 2014. Desses, dois foram selecionados, e três capítulos, utilizados. A análise dos artigos e capítulos de livros baseou-se na descrição do conteúdo dos textos associada aos objetivos do estudo.

Para a organização e apresentação dos resultados, foram definidos quatro campos temáticos: contexto de desenvolvimento da estratégia grupal, perspectiva teórica, modelagem das estratégias e o papel das estratégias grupais na assistência. A análise adotou o pressuposto de que não existem estratégias generalizáveis e descontextualizadas, e que as propostas identificadas apresentam diferentes aspectos técnicos, científicos, epidemiológicos e relacionais que devem ser apreciados e comparados.

\section{Contexto de desenvolvimento da estratégia grupal}

Os textos estudados apresentam experiências grupais dirigidas aos familiares desenvolvidas nos seguintes contextos: Centro de Atenção Psicossocial para usuários de álcool e outras drogas - CAPSad ${ }^{16,17}$, Núcleo de Atenção Psicossocial para Farmacodependentes NAPS $\mathrm{F}^{18}$, Ambulatórios vinculados a hospitais-escolas de universidades públicas ${ }^{19,20,21}$, Centro de Convivência ${ }^{22}$ e Clínica Psiquiátrica ${ }^{23}$, em diversos municípios e regiões brasileiras.

Os ambulatórios contavam com terapeutas familiares, que adequavam suas bases teóricas às especificidades das demandas. Os serviços que integram redes de saúde municipais (CAPSad, NAPS F, Centro de Convivência) ou privados (Clínica) não referiram contar com equipes específicas de terapia familiar.

Os textos mostram que os serviços de saúde mental são flexíveis ao construírem suas estratégias de atenção aos familiares: o trabalho assistencial é desenvolvido por equipes multiprofissionais que adotam a posição teórica e técnica de que o cuidado à família é essencial ao tratamento das pessoas com necessidades decorrentes do uso de substâncias psicoativas. Tal posição também é compartilhada pela opinião de familiares ${ }^{16,22,19}$.

As equipes compartilham a gestão dos grupos, de tal maneira que profissionais das áreas de psicologia, terapia ocupacional, enfermagem e serviço social são citados como responsáveis pela condução dos grupos, conforme a autoria dos textos aqui estudados. 


\section{Perspectivas teóricas}

Os textos mostram que os profissionais adotam diversas perspectivas teóricas sobre funcionamento familiar e o consumo de substâncias psicoativas, dentre elas se destacam as abordagens: cognitivo-comportamentais, sistêmicas e psicodinâmicas.

As abordagens cognitivo-comportamentais concebem a dependência como doença e combinam a oferta de informações com estratégias de enfrentamento baseadas em orientações de condutas na relação familiar e no manejo com os usuários. Visam reduzir comportamentos e padrões cognitivos compatíveis com o uso de substâncias psicoativas, reforçar aspectos que podem inibir o uso, e promover reflexões entre os familiares, a partir de estratégias psicoeducativas ${ }^{19,22,24}$.

As abordagens de orientação sistêmicas ${ }^{18,20,23}$ e psicodinâmicas ${ }^{16,17,21}$ concebem o uso prejudicial a partir de seu papel nos diferentes sistemas relacionais do usuário, sendo a família um sistema primordial nestas análises. O padrão vincular de adoecimento do núcleo familiar (codependência), a configuração da família em torno de conflitos de papéis, valores e crenças geracionais, assim como as concepções e fantasias atribuídas ao usuário de substâncias psicoativas podem ser tomados como analisadores ou promotores de insights. Estratégias de promoção do diálogo entre os participantes, com ou sem oferta de informações sobre o consumo de substâncias psicoativas, podem ampliar a reflexão e contribuir para ampliar a rede sociorrelacional.

\section{Modelagem das estratégias}

Os textos não são suficientemente explícitos em descrever e detalhar a organização das práticas grupais. No geral, limitam-se a citar a duração, a maior parte definida em uma hora, e a periodicidade, geralmente semanal. Caracteriza as diferentes abordagens a adoção da composição multifamiliar, sendo os grupos abertos à participação de familiares de diversos usuários dos serviços. Mostram que a presença das mães e membros do sexo feminino ${ }^{17,19,22}$ é marcante. Um estudo ${ }^{18}$ sugere que a oferta de grupos aconteça em diferentes horários para garantir a ampliação do acesso dos familiares.

Em relação às sessões, três estratégias descritas caracterizam-se pela continuidade definida de acordo com o desejo de participação dos familiares ${ }^{16,18,20}$. Os demais estudos propunham número de sessões pré-estabelecidos, não abertos às demandas espontâneas. Resumidamente, o primeiro ${ }^{19}$ descreve seis sessões de orientação em caráter temático $\left(1^{\mathrm{a}}\right.$, contrato e queixa; $2^{\mathrm{a}}$, trabalhar reações típicas; comportamentos indicativos de uso/recaída; $3^{\mathrm{a}}$, estágios de mudança e dinâmica da personalidade; $4^{\mathrm{a}}$, como ajudar e quais comportamentos evitar; $5^{\mathrm{a}}$, informação sobre drogas; $6^{\mathrm{a}}$, plano de ação para cada caso); o segundo ${ }^{24}$ descreve oito sessões de psicoeducação no modelo de treino de habilidades sociais ( $1^{\mathrm{a}}$, apresentação e contrato; $2^{\mathrm{a}}$, informação; $3^{\mathrm{a}}$, como agir assertivamente; $4^{\mathrm{a}}$, como manejar a raiva; $5^{\mathrm{a}}$, como falar e ouvir sobre sentimentos; $6^{\mathrm{a}}$, como fazer e receber elogios e críticas; $7^{\mathrm{a}}$, como resolver problemas; $8^{\mathrm{a}}$, feedback do tratamento e encaminhamentos); e o terceiro ${ }^{21}$ descreve sete sessões de orientação em caráter temático $\left(1^{\mathrm{a}}\right.$, apresentação geral e identificação de ciclo de vida; $2^{\mathrm{a}}$, discussão sobre situações familiares; $3^{\mathrm{a}} / 4^{\mathrm{a}} / 5^{\mathrm{a}}$, comunicação; $6^{\mathrm{a}}$, orientar e ser orientado; $7^{\mathrm{a}}$, encerramento).

Uma estratégia grupal desenvolvida no contexto de internação estabeleceu temas considerados relevantes ao contexto: fase pós-desintoxicação, início das abordagens individuais, alta hospitalar e início da ressocialização ${ }^{23}$.

Dois estudos ${ }^{16,20}$ apresentavam a estratégia grupal desenvolvida por meio de depoimentos dos participantes que dialogam e trocam experiências, com o apoio mútuo e dos profissionais participantes.

As experiências mostram a utilização de dinâmicas de grupo ${ }^{21,24}$, role playing e tarefas pós-grupo ${ }^{24}$, recursos expressivos/artísticos ${ }^{17}$, em conformidade com os objetivos e bases teóricas das estratégias.

\section{Papel das estratégias grupais na assistência}

Constituem-se enquanto objetivos da oferta de atenção grupal aos familiares: a melhora das relações entre familiares de usuários $^{19,22,24}$; o reconhecimento de aspectos da codependência visando tornar os familiares conscientes das responsabilidades e condutas possíveis de serem assumidas ${ }^{17}$; a avaliação da dinâmica familiar e observação de demandas para encaminhamentos ${ }^{18-20,24}$; e a adesão dos usuários ao tratamento ${ }^{23}$.

As estratégias grupais direcionadas às pessoas que vivem situações semelhantes podem facilitar a identificação, a troca de confidências e a ampliação do grau de intimidade entre os membros, aspectos importantes para a condução do processo grupal ${ }^{16}$ e adesão dos familiares ${ }^{24}$. A partir de experiências clínicas, considerou-se benéfico o acolhimento grupal de familiares na medida em que os participantes, ao compartilharem suas angústias ${ }^{20}$, podem tranquilizar-se, ampliar a visão sobre as situações vividas e dialogar sobre novas questões além do problema ${ }^{21}$.

Em relação aos efeitos das estratégias, um estudo analisou os motivos e a importância da participação a partir 
da opinião dos familiares, e identificou que compreendem os grupos como lugar de escuta e apoio sem julgamento, o que possibilita a expressão de sentimentos, redução do isolamento e desesperança, a troca de experiências e aprendizado sobre estratégias de enfrentamento ${ }^{16}$. Outro estudo analisou a percepção dos familiares sobre aspectos da vivência com usuários e sua participação na estratégia grupal. Observou-se que contribuem para o aprendizado de modos de enfrentar as recaídas, o entendimento da dependência como doença, o sentimento de acolhimento encontrado no grupo, a aquisição de novas amizades, aumento da autoestima e o reconhecimento de sentimentos negativos como vergonha, impaciência e desespero ${ }^{22}$.

Um estudo considera o grupo como um dispositivo para a expressão da subjetividade e sentimentos em relação à dependência, que permitiu aos participantes reconhecerem aspectos da codependência, do adoecimento diante da sobrecarga e mudanças na dinâmica familiar ${ }^{17}$. Outro estudo destacou o que para os familiares foi importante na estratégia: a aquisição de informações, as orientações dos profissionais sobre condutas em situações de intoxicação e agressividade e a troca de experiências entre participantes ${ }^{19}$.

\section{DISCUSSÃO}

\section{As estratégias grupais como dispositivos de cuidado aos familiares}

Os estudos confirmam a importância de oferecer aos familiares ações de suporte e identificam seus benefícios: identificação, reconhecimento e compreensão das próprias necessidades de saúde, sociais e emocionais, que estimulam a procura de ajuda; ampliação da compreensão sobre a problemática enfrentada, que contribui na redução da ansiedade e na desmistificação do comportamento dos usuários de drogas; a redução do nível de tensão familiar por meio da melhora na comunicação; redução do isolamento e oferta de informações sobre serviços e redes; aumento do suporte para o usuário de drogas, na medida em que as famílias adquirem habilidades para lidar com os problemas ${ }^{8}$. Considera-se, entretanto, uma tendência a marginalizar a família e as redes sociais dos usuários na assistência ${ }^{25}$.

Segundo Yasui ${ }^{26}$ o cuidado em saúde "significa pensar em uma atitude de ocupação, preocupação, de responsabilização e envolvimento com o outro". Acolher usuários e familiares e torná-los sujeitos requer a mudança no olhar e na escuta profissional, e a ruptura com a racionalidade médica que reduz as pessoas a objetos de intervenção. Deve-se acolher os sujeitos e buscar compreender suas histórias e singularidades, de tal forma que as "estratégias de intervenção daí decorrentes ampliam-se para a construção de projetos marcados pela diversidade. Cuidar é montar, tecer projetos de vida" ${ }^{26}$.

Qualificar o cuidado implica a reflexão acerca dos aspectos relacionais, técnicos e éticos na composição das ofertas de estratégias grupais, por meio de tecnologias em saúde ${ }^{10,11}$ baseadas no desenvolvimento de relações possíveis a partir do encontro entre equipe, usuários, familiares e comunidade, e que requerem ancoragem na formação teórica e técnica dos profissionais nos campos de saberes comuns e específicos.

Quando se encontram em situações vulneráveis, as famílias podem ter muitas expectativas em relação ao tratamento do usuário ou ao suporte familiar. Considerando o aspecto relacional entre familiares e profissionais, e sua importância enquanto tecnologia leve de cuidado, devese observar os riscos presentes nas ações, geralmente envolvidos com as habilidades dos profissionais que podem comprometer a autonomia, corresponsabilização, a produção de vínculo e acolhimento.

Cechin $^{27}$ e Rosa ${ }^{7}$ identifica os riscos presentes em cinco tipos de respostas presentes no trato com familiares: a) a necessidade de ser útil, o que pode colocar a família no lugar de impotência, já que não constrói novas soluções para seus problemas; b) o aconselhamento professoral, que tende a diminuir a qualidade interacional entre profissional e familiar e as possibilidades de aprendizado; c) o controle excessivo do grupo, que pode tornar os membros apáticos e dependentes; d) a necessidade de proteção daqueles que julga desestruturados, infeliz ou desorganizados, que pode fazer que o profissional tome para si a tarefa de reorganização familiar, o que reduz a capacidade dos familiares na resolução de situações; e) a punição, na qual o profissional dá lições a quem considera ter comportamento inadequado.

As famílias, ao buscarem tratamento ou ao acompanharem um familiar ao serviço, tanto incentivam ou controlam a aderência ao tratamento quanto explicitam demandas às equipes em relação às recaídas e orientação sobre condutas cotidianas. Outras necessidades, referidas pelos familiares, se apresentam como queixas difusas, que envolvem seus modos de vida: situações de isolamento social, com abolição de situações de prazer e interação; desengajamento em atividades profissionais, educacionais, religiosas, sociais e planos de vida; mudanças na rotina e nos papéis familiares; e sentimentos de desamparo, desesperança e culpa, que podem inviabilizar reflexões sobre a multidimensionalidade de fatores associados ao desenvolvimento e manutenção de uso prejudicial de substâncias psicoativas. 
As estratégias psicoeducativas contribuem com oferta de informações relativas aos múltiplos fatores envolvidos no abuso ou dependência de substâncias psicoativas, procurando deslocar crenças que favorecem preconceitos, rótulos e estigmas e que não contribuem para o resgate da autonomia ${ }^{22}$. Visam à melhora nas relações familiares por meio de habilidades sociais, resolução de problemas e condutas de ação nas situações de intoxicação e agressividade. As estratégias de apoio e ampliação de rede sócio relacional podem possibilitar a expressão de sentimentos negativos e ambivalentes associados à vivência cotidiana com os usuários, reconstruindo espaços de sociabilidade que facilitam a interação, a troca e a abertura de narrativas marcadas pelo sofrimento, pela vergonha e desorientação que acompanham a história de vida de familiares, e a ação de cuidado sobre vetores que vulnerabilizam e fragilizam a própria vida dos familiares.

As estratégias grupais dirigidas aos familiares apresentam potencial de cuidado, pois respondem às necessidades de ampliação do acesso das famílias aos serviços e de compreensão sobre uso prejudicial de substâncias psicoativas e seus tratamentos. Fortalecem estratégias de educação em saúde e promovem aproximação e vínculo entre profissionais, familiares e usuários, ofertando suporte social e emocional, a partir do estabelecimento de redes de relações solidárias e de cuidado. Entretanto deve-se ter prudência na montagem de estratégias, para que se evite a reprodução de técnicas

\section{REFERÊNCIAS}

1. Amarante P. Saúde mental e atenção psicossocial. Rio de Janeiro: Fiocruz; 2007.

2. Thornicroft G, Tansella M. Boas práticas em saúde mental comunitária. Barueri: Manole; 2010.

3. Barroso SM, Bandeira M, Nascimento E. Sobrecarga de familiares de pacientes psiquiátricos atendidos na rede pública. Rev Psiq Clín. 2007;34(6): 270-7. Disponível em: http://www.hcnet.usp.br/ipq/revista/vol34/n6/270.html.

4. Schenker M, Minayo MCS. A importância da família no tratamento do uso abusivo de drogas: uma revisão da literatura. Cad Saúde Pública. 2004;20(3):649-59. doi: http:// dx.doi.org/10.1590/S0102-311X2004000300002.

5. Souza MS, Baptista MN. Associações entre suporte familiar e saúde mental. Psicol Argum 2008;26(54):20715. Disponível em: http://www2.pucpr.br/reol/index.php/ PA?dd1 $=2495 \&$ dd99= view. descontextualizadas dos serviços e das necessidades de familiares.

\section{CONSIDERAÇÕES FINAIS}

$\mathrm{Na}$ atualidade, o desenvolvimento de estratégias psicoeducativas e de apoio e ampliação de rede sócio relacional é a principal ancoragem teórica e técnica adotada pelos serviços de saúde mental na atenção aos familiares de pessoas com necessidades decorrentes do uso de substâncias psicoativas. A potencialidade dessas estratégias ao responderem às necessidades de saúde são relevantes. A escolha e a adoção dos referenciais técnicos e teóricos devem ocorrer no contexto de processos reflexivos e considerar as necessidades apresentadas pelos familiares; reconhecer as histórias de vida e singularidades dos sujeitos; e priorizar a produção de vínculos e autonomia.

Essa perspectiva, ao invés de reproduzir técnicas grupais descontextualizadas, amplia o pensamento estratégico do cuidado em Saúde Mental e coloca em diálogo os saberes das profissões de saúde, as diretrizes do Sistema Único de Saúde (SUS) e da Reforma Psiquiátrica, as necessidades das famílias e a dimensão relacional do trabalho em saúde.

Espera-se que este estudo possa subsidiar discussões nos serviços de saúde mental quanto à montagem de estratégias de cuidado voltadas aos familiares.

6. Brasil. Ministério da Saúde. A política do Ministério da Saúde para a atenção integral a usuários de álcool e outras drogas. Brasília: Ministério da Saúde; 2004. Disponível em: http://bvsms.saude.gov.br/bvs/publicacoes/pns_alcool drogas.pdf.

7. Rosa LCS. A família como usuária de serviços e como sujeito político no processo de reforma psiquiátrica brasileira. In: Vasconcelos EM, organizador. Manual de ajuda e suporte mútuos em Saúde Mental para facilitadores, trabalhadores e profissionais de saúde e saúde mental. Rio de Janeiro: UFRJ/ Ministério da Saúde; 2013. Disponível em: http://www.crprj. org.br/documentos/noticia2014_070214_02.pdf.

8. Scotland. The Scottish Government. EIU (Effective Interventions Unit). Supporting families and carers of drug users: a review. Edinburgh: Scottish Executive; 2002. Disponível em: http://www.gov.scot/Resource/ Doc/47007/0025628.pdf. 
9. Cecílio LCO. As necessidades de saúde como conceito estruturante na luta pela integralidade e equidade na atenção à saúde. In: Pinheiro R, Mattos RA, organizadores. Os sentidos da integralidade na atenção e no cuidado à saúde. Rio de Janeiro: IMS/UERJ/Abrasco; 2001.

10. Merhy EE. Em busca de ferramentas analisadoras das tecnologias em saúde: a informação e o dia a dia de um serviço, interrogando e gerindo trabalho em saúde. In: Merhy EE, Onocko R, organizadores. Agir em saúde: um desafio para o público. São Paulo: Hucitec; 1997.

11. Jorge MSB, Pinto DM, Quinderé PHD, Pinto AGA, Sousa FSP, Cavalcante CM. Promoção da saúde mental - tecnologias do cuidado: vínculo, acolhimento, corresponsabilização e autonomia. Cien Saúde Coletiva. 2011;16(7):3051-60. doi: http://dx.doi.org/10.1590/S141381232011000800005 .

12. Vasconcelos EM, organizador. Manual de ajuda e suporte mútuos em saúde mental para facilitadores, trabalhadores e profissionais de saúde e saúde mental. Rio de Janeiro: UFRJ/ Ministério da Saúde; 2013. Disponível em: http:/www.crprj. org.br/documentos/noticia2014_070214_02.pdf.

13. Brasil. Ministério da Saúde. Saúde mental no SUS: os centros de atenção psicossocial. Brasília: Ministério da Saúde; 2004. Disponível em: http://www.ccs.saude.gov.br/saude_mental/ pdf/sm_sus.pdf.

14. Tavares EC. Sobre revisão narrativa, integrativa e sistemática. Belo Horizonte, 2010. Disponível em: http://www.nescon. medicina.ufmg.br/agora.

15. Rother ET. Revisão sistemática $\mathrm{x}$ revisão narrativa [editorial]. Acta Paul Enf. 2007;20(2):v-vi. doi: http://dx.doi. org/10.1590/S0103-21002007000200001.

16. Alvarez SQ, Gomes GC, Oliveira AM, Xavier DM. Grupo de apoio/suporte como estratégia de cuidado: importância para familiares de usuários de drogas. Rev Gau Enf. 2012;33(2):102-8. doi: http://dx.doi.org/10.1590/S198314472012000200015 .

17. Moraes LMP, Braga VAB, Souza AMA, Oriá MOB. Expressão da codependência em familiares de dependentes químicos. REME Rev Min Enf. 2009;13(1):34-42. doi: http://www.dx.doi.org/S1415-27622009000100005.

18. Moreira MSS. A dependência familiar. Rev SPAGESP. 2004;5(5):83-8. Disponível em: http://pepsic.bvsalud.org/ pdf/rspagesp/v5n5/v5n5a15.pdf.

Recebido para publicação: 07.05.15

Aceito para publicação: 04.06.15
19. Figlie NB, Pillon SC, Dunn J, Laranjeira R. Orientação familiar para dependentes químicos: perfil, expectativas e estratégias. J Bras Psiq. 1999;48(10):471-8. Disponível em: http://uniad. org.br/desenvolvimento/images/stories/pdf/Orientacao Familiar_para_DQ-_J_Bras_Psiq 4810471-478_1999.pdf.

20. Dualib C. Acolhimento para familiares de dependentes: um olhar sistêmico no trabalho com Famílias. In: Silveira DX, Moreira FG, organizadores. Panorama atual de drogas e dependências. São Paulo: Atheneu; 2006.

21. Maluf TPG, Pires EKP. Orientação familiar: uma perspectiva do ciclo de vida familiar. In: Silveira DX, Moreira FG. Panorama atual de drogas e dependências. São Paulo: Atheneu; 2006.

22. Matos MTS, Pinto FJM, Jorge MSB. Grupo de orientação familiar em dependência química: uma avaliação sob a percepção dos familiares participantes. Rev Baiana Saúde Publica. 2008;32(1):58-71. Disponível em: http://inseer. ibict.br/rbsp/index.php/rbsp/article/viewFile/1384/1020.

23. Seadi SMS, Oliveira MS. A terapia multifamiliar no tratamento da dependência química: um estudo retrospectivo de seis anos. Psicol Clin. 2009;21(2):363-78. doi: http:// dx.doi.org/10.1590/S0103-56652009000200008.

24. Jungerman FS, Zanelatto N. Tratamento do usuário de maconha e seus familiares: um manual para terapeutas. São Paulo: Rocca; 2007.

25. Barnard M. Drugs in the family: the impact on parents and siblings. Glasgow: Joseph Rowntree Foundation, University of Glasgow; 2005.

26. Yasui S. Rupturas e encontros: desafios da reforma psiquiátrica brasileira [Tese]. Rio de Janeiro: Escola Nacional de Saúde Pública, Fiocruz; 2006. p.112.

27. Cecchin G. Sistemas terapêuticos e terapeutas. In: Elkaim M. Terapia familiar em transformação. São Paulo: Summus Editorial; 2000. p. 72-77. Apud Rosa LCS. A família como usuária de serviços e como sujeito político no processo de reforma psiquiátrica brasileira. In: Vasconcelos EM, organizador. Manual de ajuda e suporte mútuos em saúde mental para facilitadores, trabalhadores e profissionais de saúde e saúde mental. Rio de Janeiro: UFRJ/Ministério da Saúde; 2013. p.145-6. Disponível em: http://www.crprj.org. br/documentos/noticia2014_070214_02.pdf. 